\title{
Miocardiopatía por hipocalcemia
}

Ronald Johnson, ${ }^{1}$ Jorge Toloza, ${ }^{2}$ Lorena Cortes, ${ }^{3}$ Cristian Valdés. ${ }^{4}$

Unidad de Paciente Crítico Adultos - Hospital Regional E. Torres G., Iquique.

4) Alumno de Medicina. Universidad del Mar, Sede lquique

\section{Resumen:}

Se presenta el caso de un varón de 56 años, portador de Miocardiopatía dilatada, considerada, en algún momento, como terminal. Se comprobó que presentaba de base una hipocalcemia crónica secundaria a hipoparatiroidismo. Se discute su notable respuesta a administración de calcio e infusión de levosimendan IV fármaco inótropo positivo que actúa sensibilizando al calcio.

\section{Caso clínico}

FC 23456

Paciente hombre de 54 años, el año 2005 estando asintomático se constata cardiomegalia en Rx tx ,durante una evaluación preoperatoria para intervención de cataratas. Se realiza ecocardiograma que mostró dilatación biventricular, signos de hipertensión pulmonar, dilatación auricular izquierda, insuficiencia mitral moderada y compromiso importante de la fracción de eyección, confirmando una miocardiopatía Dilatada (figuras 1 y 2)

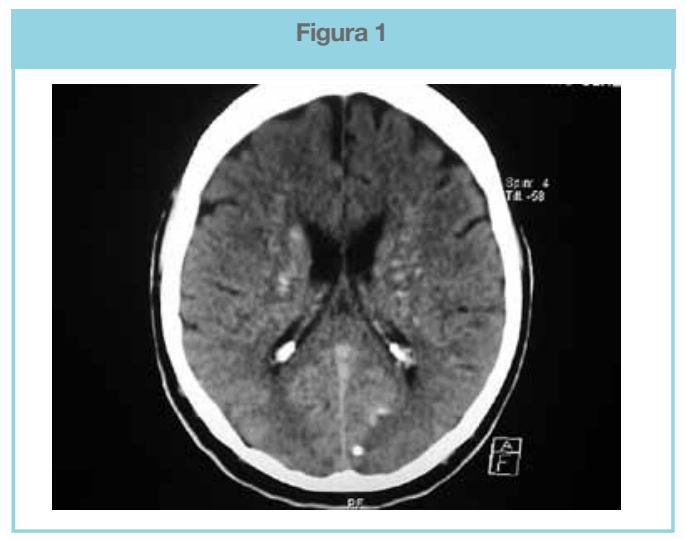

Correspondencia:

Ronald Johnson Q.

Barros Arana 1550 Iquique

Fono 57-319106

correo electrónico:rjohnsonq@gmail.com
Evoluciona sintomático, con deterioro progresivo de la capacidad funcional, requiriendo hospitalizaciones frecuentes durante el año 2005 (en cuatro oportunidades).

En febrero del 2006 se le realizó estudio coronariográfico que descartó enfermedad coronaria. Ese año es hospitalizado en forma reiterada (6 veces), evaluado como terminal, se le plantea la posibilidad de trasplante cardíaco, recibiendo infusiones de Dobutamina IV. En Mayo durante una hospitalización se detecta Fibrilación auricular y niveles bajos de calcemia iniciando terapia con gluconato de calcio IV.

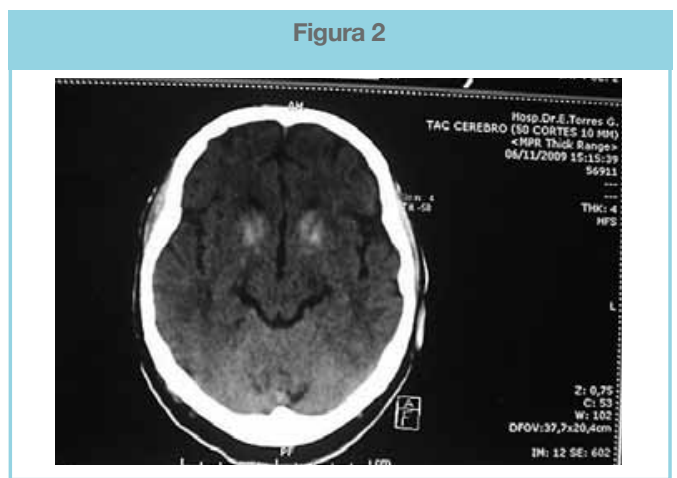




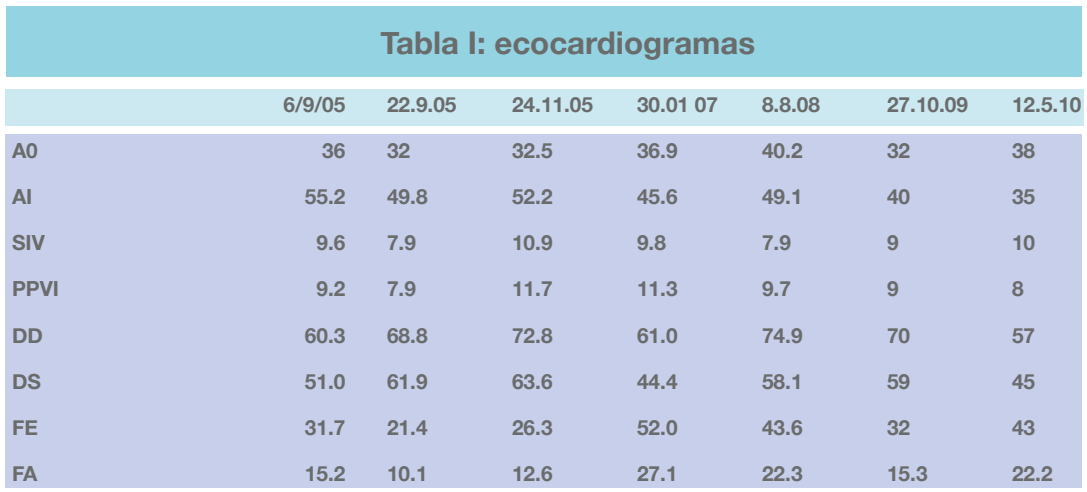

Durante hospitalización en Junio de ese año presenta hipotensión mialgias severas y oliguria. Se decide tratar con infusión de levosimendan $2.5 \mathrm{mg}$., también recibió calcio IV. Destaca una respuesta rápida y espectacular con mejoría importante de su sintomatología.

Luego del alta, fue enviado a Santiago para evaluación de trasplante. El estudio reveló un gasto cardíaco de 4,5 lts/min con consumo de $\mathrm{O} 2 \mathrm{de} 18 \mathrm{ml} / \mathrm{Kg} / \mathrm{min}$ y $\mathrm{PCWP}$ de $17 \mathrm{mmHg}$ y se considera que aún no cumple criterios para ser sometido a esta cirugía. La evolución es a la mejoría y durante el año 2007 y 2008 no requirió hospitalizaciones. El estudio ambulatorio ecocardiografico durante ese periodo demostró mejoría de la función ventricular (tabla I). Reingresa en Enero del 2009 a raíz de descompensación con disnea CF IV y Síndrome de QTlargo (figura 3). Es compensado y se da alta con IECa, diuréticos, espironolactona y carvedilol.

Luego de algún tiempo, reaparecen manifestaciones de disfunción ventricular y episodios sincopales y convulsivos, con severo trauma facial. Se confirmaron arritmias ventriculares asociadas a QT prolongado (figura 4) en el contexto de hipocalcemia severa de $3,9 \mathrm{mg} \%$ con fosfemia de $9,9 \mathrm{mg} \%$. Los niveles de PTH bajos $8 ; 1 \mathrm{pg} / \mathrm{ml}$ confirmaron hipoparatiroidismo por lo que se administró cal-

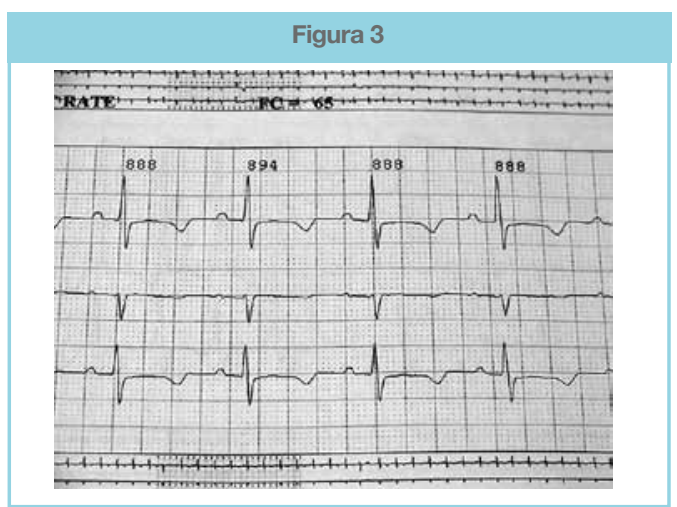

cio y vit D. El ECG mostró una rápida regresión del QT y mejoría en su capacidad funcional. En Septiembre del 2009 es enviado a Antofagasta donde se le implanta MDI.

Reingresa al mes siguiente por nuevo episodio sincopal convulsivo. Se realiza determinación de calcemia de 3.2. SSe completa estudio con Tac cerebral que revela calcificaciones de ganglios basales (figuras 5 y 6). El paciente había suspendido terapia con calcitriol, el que fue reiniciado, mejorando su capacidad funcional hasta $\mathrm{CFI}$, con mejoría objetiva de su función ventricular a ECO.
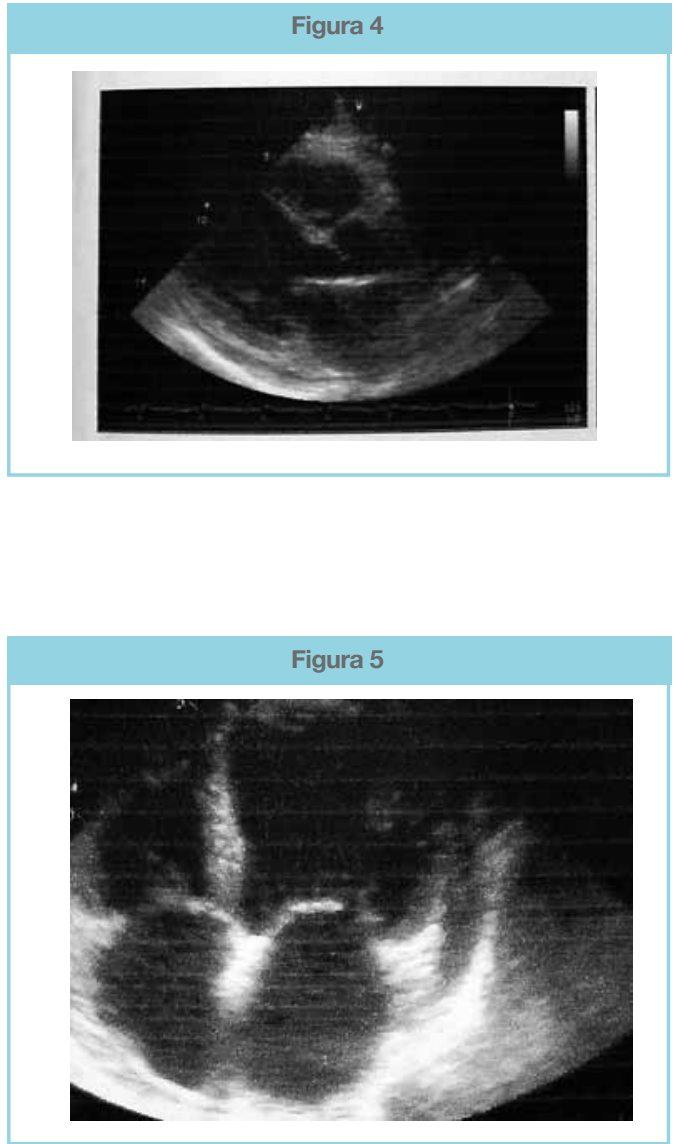


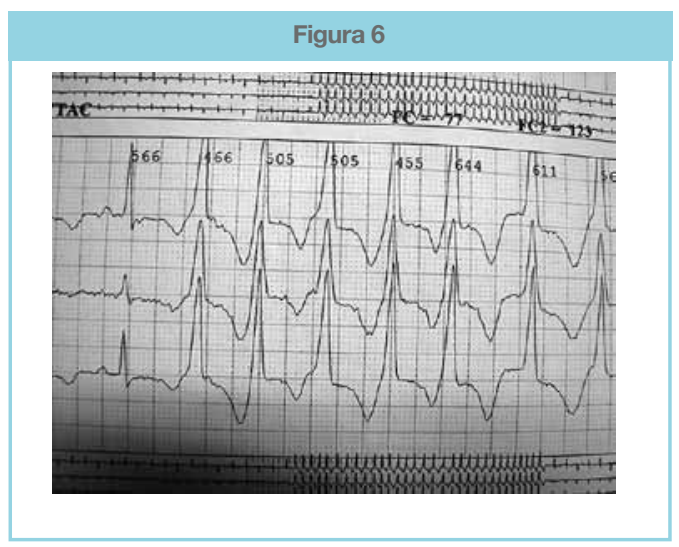

\section{Discusión:}

El calcio intracelular y su unión a troponina $\mathrm{C}$ es un elemento central en el mecanismo de contracción y relajación de las células miocárdicas ${ }^{1}$. La contracción de la fibra muscular se debe a la interacción de las proteínas actina y miosina, regulada por la entrada de calcio desde el medio extracelular durante la activación de la célula cardíaca. En este contexto, la hipocalcemia ejerce un efecto inotrópico negativo sobre las fases de contracción-excitación provocando disfunción progresiva tanto sistólica como diastólica y la conducción cardíaca, con una característica prolongación del intervalo QT del ECG ${ }^{2-3}$. Por otro lado, la paratohormona tiene un efecto inótropo positivo al aumentar la entrada de calcio a los miocitos y liberación de noradrenalina endógena miocárdica. Así el hipoparatiroidismo con o sin hipocalcemia puede llevar a insuficiencia cardíaca refractaria a terapia convencional ${ }^{4}$.

Se ha descrito, ya por varios autores ${ }^{5-9}$, la presencia de insuficiencia Cardíaca Congestiva por disfunción sistólica y diastólica en relación a hipocalcemia crónica, condición que ha demostrado reversibilidad al administrar calcio y vitD en forma mantenida ${ }^{10-14}$.

En nuestro paciente el diagnóstico se evidenció luego de varias hospitalizaciones por IC Descompensada, en los que siempre ingresaba con hipocalcemia. Su consulta inicial, asintomático con Rx Tórax que mostró cardiomegalia fue a raíz de una evaluación preoperatoria de cataratas, patología descrita en pacientes con hipoparatiroidismo ${ }^{15-16}$. El paciente recibió calcio en cada una de las hospitalizaciones, mostrando mejoría clínica, incluso mantuvo un período de 2 años sin requerir nuevos ingresos, con controles ambulatorios esporádicos. En este lapso de tiempo no recibió calcio, reiniciando su sintomatología con reaparición de disfunción VI y arritmias secundarias a QT prolongado.

El estudio de su patología de base ha demostrado un hipoparatiroidismo con calcemias persistentemente bajas, asociadas a arritmias y episodios convulsivos y sincopales algunos de los cuales pueden ser atribuidos a arritmias ventriculares demostradas en estudio holter y otras a convulsiones propias de su cuadro basal.

El estudio cerebral con TAC demostró calcificaciones de los ganglios basales, condición descrita en la literatura ${ }^{17,18}$ y que se manifiesta con tal sintomatología. Las calcificaciones cerebrales en el hipoparatiroidismo se han relacionado con crisis comiciales, síntomas extrapiramidales (coreoatetosis y parkinsonismo), cerebelosos (ataxia, disartria) y trastornos neuropsiquíatricos (ansiedad, depresión, labilidad emocional, retraso mental, demencia, psicosis) ${ }^{19}$, siendo difícil establecer en cada caso si estas manifestaciones neurológicas se deben a las alteraciones electrolíticas del hipoparatiroidismo (hipocalcemia, hiperfosfatemia, hipomagnesemia) o a la afectación estructural secundaria a las calcificaciones cerebrales, como en la enfermedad de Fahr ${ }^{20}$

Cabe destacar que una de las medidas más significativas en su terapia fue haber recibido infusión IV de levosimendan, lo que a la luz de los hallazgos posteriores, se explica al recibir un sensibilizador al calcio junto con aporte de calcio administrado IV a raíz de hipocalcemia demostrada en ese instante. Levosimendán potencia la sensibilidad al calcio de los miofilamentos al unirse a la troponina $\mathrm{C}$ cardíaca, por un mecanismo dependiente del calcio, lo cual modifica la cinética de uniones cruzadas de la actina miosina sin afectar aparentemente a la tasa cíclica de uniones cruzadas ni al consumo miocárdico de ATP ${ }^{21}$. Aumenta la fuerza de contracción, pero sin afectar a la relajación ventricular, debido a la menor afinidad de unión cuando las concentraciones citosólicas de calcio se reducen durante la diástole.

La demostración de arritmias ventriculares en el contexto de una Miocardiopatía Dilatada, llevó a la implantación de un MDI, dispositivo que a nuestra consideración ha prestado claros beneficios al descargar frente a arritmias ventriculares en periodos que cursó con hipocalcemia severa. 


\section{Referencias:}

1. Schlant RC. Normal physiology of the cardiovascular system. In: Hurst JW, ed. The heart, 4th ed. New York: McGraw-Hill, 1978; $71-73$

2. Connor TB, Rosen BL, Blaustein MP,Applefeld MM,Doyle LA. Hypocalcemia precipitating heart failure. N Engl J Med 1982; 307: 869-872,

3. Morgan JP.Abnormal intracellular modulationof calcium as a major cause of cardiac contractile dysfunction. N Engl J Med 1991; 325: 625-632.

4. Suzuki T, Ikeda U, Fujikawa H, Saito K, Shimada K. Hypocalcemic heart failure: a reversible form of heart muscle disease. Clin Cardiol 1998; 21: 227-8.

5. Altunbas H, Balci M, Yazicioglu G, Semiz E, Ozbilim G, Karayalcin U. Hypocalcemic cardiomyopathy due to untreated hypoparathyroidism. Horm Res 2003; 59: 201-4.

6. Gulati S, Bajpai A, Juneja R, Kabra M, Bagga A, Kalra V. Hypocalcemic heart failure masquerading as dilated cardiomyopathy. Indian J Pediatr 2001; 68: 287-90.

7. Greca A, Hernández N, Ríos R, Settecase C, Villa L, Libman J et al. Cardiac failure secondary to hypoparathyroidism. An unusual presentation. Medicina Buenos Aires 1997; 57: 320-2.

8. Takaaki S, Uichi I, Hideyjuki F, Ken S, Kazuyuki S. Hypocalcemic Heart Failure: A Reversible Form of Heart Muscle Disease. Clin Cardiol 1998; 21: 227-8.

9. Monig H, Foh KP, Schulte HM, Simon R. Hypocalcemic cardiomyopathy as the cause of severe left heart failure. Dtsch Med Wochenschr 1994; 119: 1270-5.

10. Mikhail N, El-Bialy A, Grosser J. Severe hypocalcemia: a rare cause of reversible heart failure. Congest Heart Fail 2001; 7: 256-8.

11. Chraibi S, Drighl A, Nafidi S, Zahraoui M, Tahiri A, Chraibi N. Hypocalcemic dilated cardiomyopathy: rare cause of heart failure.
Ann Med Interne (Paris). 2001; 152: 483-5.

12. Avsar A,Dogan A.,Tavli T.A rare cause of reversible Dilated myocardiopathy:hypocalcemia. Ecocardiography,2004. 609-12

13. Chraibi S.,Dight A,Nefidi S,Zahraoui M,Tahiri A,Chraibi N.Hypocalcemic Dilated myocardiopahy:Arare cause of Heart Failure ,Ann Med Interne(Paris).2001; 152: 483-5

14. Małek M, Elikowski W. [Hypocalcemic cardiomyopathy--a reversible type of cardiac failure in a patient with primary hypoparathyroidism and coexisting Fahr's disease]. Kardiol Pol. 2003; 58 : 129-34.

15. Ardouin M, Dary J, Murie N, Belloir J, Chesnais A. Eye manifestations in phospho-calcic metabolic disorders. Cataract in the course of hypoparathyroidism. Arch Ophtalmol Rev Gen Ophtalmol. 1972; 32: 71-4.

16. POHJOLA S. Ocular manifestations of idiopathic hypoparathyroidism. Acta Ophthalmol (Copenh). 1962; 40: 255-65.

17. Calvo JM. Calcificaciones cerebrales múltiples asintomáticas y el eje vitamina D-hormona paratiroidea. Rev Neurol 2001; 32: 1198-9.

18. Jover-Díaz F, Pomares F, Matarranz M. Calcificaciones intracranealesextensas en el hipoparatiroidismo crónico. Rev Neurol 2006; 42: 61-2.

19. Sachs CH, Sjöberg HE, Ericson K. Basal ganglia calcifications on CT: Relation to hypoparathyroidism. Neurology 1982; 32: 779-82.

20. Cartier L, Passig C, Gormaz A, Lopez J.Cambios neuropsicológicos y neurofisiológicos en la enfermedad de Fahr. Rev Med Chile 2002; 130: 1283-90.

21. Despas F, Trouillet C, Franchitto N, Labrunee M, Galinier M, Senard JM, et al. Levosimedan improves hemodynamics functions without sympathetic activation in severe heart failure patients: Direct evidence from sympathetic neural recording. Acute Card Care. 2010; 12: 25-30. 